\title{
Association of stroke severity with functional outcome using National Institute of Health (NIH), Functional Independence Measurement(FIM) and Stroke Specific Quality of Life (SSQOL) in stroke patients
}

\author{
Ragini Zatale ${ }^{1}$, Snehal Joshi ${ }^{2, *}$, Hiral Soni ${ }^{3}$ \\ ${ }^{1,3}$ M.P. Th, ${ }^{2}$ Professor, DES Brijlal Jindal College of Physiotherapy, Pune, Maharashtra, India
}

*Corresponding Author:

Email: drnehalmandke@gmail.com

\begin{abstract}
Introduction: A primary concern immediately after stroke for patients, their relatives, and their caregivers is the prospect for recovery. Several prognostic factors have been identified for outcome after stroke. However, there is a need for empirically derived STUDYs that can predict outcome and assist in medical management during rehabilitation.

This study was conducted to find if assessment of stroke severity can predict functional outcome and quality of life in patients receiving rehabilitation.

Material and Methods: NIH, FIM and SSQOL scales were evaluated on day 1, at the end of 1 month and at the end of 3 months. Scores were recorded and correlated with each other. Analysis of collected data was done using spearman's correlation.

Results: There was a statistically significant correlation between NIH, FIM and SSQOL

Conclusion: The results of the study show that there is a highly significant correlation of NIH with FIM and SSQOL. Thus, stroke severity is highly associated with functional outcome and quality of life.
\end{abstract}

Keywords: Stroke, NIH, SSQOL, FIM.

\section{Introduction}

Stroke is defined by(WHO) as a condition characterized by rapidly developing symptoms and signs of a focal brain lesion with symptoms lasting for more than 24 hours or leading to death with no apparent cause other than that of vascular origin. ${ }^{1}$

Stroke is a third leading cause of mortality worldwide and a major cause of disability. In 2009, around 10,000 people suffered from stroke in India. ${ }^{2}$

Stroke or cerebrovascular accident (CVA) is the rapid loss of brain function due to disruption in the blood supply to the brain which can be ischemic or hemorrhagic. ${ }^{1}$

Stroke is classified by etiological categories (thrombosis, embolus or hemorrhage), specific vascular territory (ACA, MCA), so forth and management categories as (TIA) minor stroke, major stroke, deteriorating stroke and young stroke. As a result, the affected area of the brain cannot function leading to inability to understand or formulate speech, or an inability to see one side of visual field, emotional liability and motor loss. Clinically, a variety of focal deficits are possible, including changes in the level of consciousness and impairment of sensory, motor, cognitive, perceptual, language function, postural control, balance, bowel and bladder functions, abnormal tone and reflexes. In addition to physical, emotional, and social consequences the economic impact of stroke is tremendous.

The location and extent of brain injury, amount of collateral blood circulation and acute care management determine the severity of neurological deficits in a stroke patient.
Stroke can result in survival with the permanent sequelae impairing in physical, psychological, and social functions. Dependence in activities of daily life living, alteration of emotional and psychological status, and deterioration in social communication can influence the Quality of life (QOL) of patients with stroke.

Rehabilitation medicine focuses on the impact of division rather than on the disease itself, therefore disability and handicap assessment is a key element in the process of rehabilitation as stated in a book "measurement in neurological rehabilitation "where importance of rehabilitation Is stressed upon while discussing assessment. ${ }^{3}$

The term measurement and assessment are used interchangeably most of the times especially when referring as tools used for collecting information. In rehabilitation, assessment refers to the process of evaluating a patient problem including recognition and measurement of problem and determining the cost and the extent. Measurement is to quantify and to determine the extent of something by comparison with a standard unit.

According to Wade there are several reasons why a patient should be assessed.

1. Diagnosis: Refers to understanding of whether a specific item is present or absent but also to the structures, activities and participation, which are impaired.

2. Prognostics: Determining who is likely to recover well and the extent to help the patient will need.

3. Measurement: Determining the severity of problem the changes that occur through time. 
4. Process: Keeping record of treatment given to patients.

5. Others: Administration and legal reasons.

The importance of using quantified measurement as part of whole assessment is to detect change, quantify input and outcome and to evaluate effectiveness of the intervention. It is also believed that by using quantified measurements the patients and relatives can be shown their improvement, thereby motivating them.

Most patients who survive a stroke, experience some physical recovery to a certain limit. Selection of the appropriate outcome measure to assess physical recovery becomes difficult, given the heterogeneity of stroke etiology, symptoms, severity, and even recovery itself. All outcome measures selected should also have acceptable psychometric properties. It is also important to establish the purpose of the measurement (discriminative, predictive, or evaluative) and to determine whether the purpose of the study is to evaluate the efficacy or effectiveness of an intervention. In addition, when selecting outcome measures and time of assessment, the natural history of stroke and stroke severity must be taken into consideration. Finally, methods for acquiring data must also be considered. So, a comprehensive overview of outcome measured should be taken.

Stroke is heterogeneous in type and severity. To characterize probabilities of outcomes and plan for rehabilitation, there is a need of a stroke scale that is able to ascertain the precise nature of stroke-related impairment and to characterize stroke severity. A good stroke scale identifies neurological impairments and is quantified so that the patient's progress can be objectively monitored. It should provide a logical basis for treatment and predict future functional outcomes. Previous researchers have demonstrated that structural and functional impairments are strongly associated with functional outcomes, but they only partially explain stroke-related disability. Nevertheless, a baseline stroke impairment scale can be used to assess stroke severity and to adequately predict functional outcome. ${ }^{4-7}$

A composite measure derived from the score of several scales like Barthel index, FIM (Functional independence measure), NIH (National institute of health), and SSQOL (Stroke specific quality of life) seems to be useful in measuring the multiple dimensions of outcomes after stroke. The NIH Stroke Scale is used to measure stroke severity at baseline. The Functional independence measure of activities of daily livings is used to measure basic activities of daily living, and SSQOL measured the patients' higher level of physical functioning and quality of life.

\section{National Institute of Health Stroke Scale (NIH)}

The National Institutes of Health Stroke Scale (NIHSS) is an assessment tool that provides a quantitative measure of stroke-related neurologic deficit.

Scores $\geq 20$ have been associated with symptomatic intracerebral hemorrhage (NINDS t-PA Stroke Study Group, 1997). NIHSS scores have been shown to strongly predict outcome after stroke and therefore can help guide decisions related to aggressiveness of care and disposition. After the initial assessment and determination of acute treatment, the focus of assessment shifts to monitoring for neurological changes or deterioration. The NIHSS provides a numerical value for comparison from one-time period to the next.

During a stroke patient's hospitalization, the NIHSS can also be used to help identify clinical findings that might put the patient at risk for complications. For instance, dysarthria and facial weakness can indicate that the patient may have difficulty swallowing. Identification of motor weakness and ataxia can alert the staff to fall risk. The stroke scale is valid for predicting lesion size and can be used as a measure of stroke severity. The NIHSS has been shown to be a predictor of both short and long term outcome of stroke patients. ${ }^{8}$

The FIM was designed to measure physical and cognitive disability and focuses on burden of care. The main objective in its development was to create a generic measure that could be administered by clinicians and non-clinicians to assess patients in all age groups with a wide variety of diagnoses Higher scores on the FIM denote patients that have a higher level of independence and require a small amount of assistance. ${ }^{9}$ The sum of all 18 items gives the patient's total score, which ranges from 18-126. ${ }^{(9)}$ The minimum score on the FIM is 18, which indicates a low level of functioning, and the maximum score is 126 , which indicates a very high level of functioning. The correlation coefficient of the inter-rater reliability ranges between 0.83 and 0.99 , and the test-retest reliability ranges between 0.84 and $0.939 .{ }^{10}$

\section{Stroke Specific Quality of Life (SSQOI)}

The SS-QOL, which is a disease-specific QOL measure, consists of 49 items encompassing 12 domains (social role, mobility, energy, language, selfcare, mood, personality, thinking, upper-extremity function, family role, vision, and work/productivity). Each item is ranked on a 5-point Likert scale. The summary score of this scale is the weighted the 12 domains. The total score ranges from 49 to245, with higher scores indicating better QOL.

Dependence in activities of daily life living, alteration of emotional and psychological status and deterioration in social communication can influence the QOL of patients with stroke. Various studies have shown a reduced QOL among patients with stroke compared with healthy individuals. Physical limitations have been stated as a determinant of the QOL. ${ }^{11}$ 
In addition to improving survival, the treatment of stroke is important for the patients' good quality of life (QOL) and thus the ultimate aim of rehabilitation of stroke patients is to improve their quality of life.

Most patients with stroke experience role changes due to impaired autonomy caused by difficulty with performing daily living activities, as well as problems with interpersonal relationships. They also face psychosocial maladjustment due to long-term stress and strain, which reduces their subjective evaluation ability of their QOL.

There are no generally accepted guidelines that determine the optimal timing, intensity or duration of rehabilitation. Many stroke patients fail to resume full lives, and a major negative impact of stroke on family is not an infrequent phenomenon. Their stroke rehabilitation requires a long term perspective, extending to several years after the onset of stroke. A primary concern immediately after stroke for patients, their relatives, and their caregivers is the prospect for recovery. Several prognostic factors have been identified for outcome after stroke. However, there is a need for empirically derived studys that can predict outcome and assist in medical management during rehabilitation.

This study was conducted to find if assessment of stroke severity can predict functional outcome and quality of life in patients receiving rehabilitation.

Aim of the study was to study association of stroke severity with functional outcome using NIH, FIM, and SSQOL in acute stroke patients.

\section{Material and Methods}

Type of study: Longitudinal Design (Observational Prospective /Cohort)

Study population: Stroke Individuals

\section{Inclusion criteria:}

1. Stroke patients on the first day

2. Patients on medications and physiotherapy treatment

3. Patients of both genders

4. Willingness by participants/ relatives.

5. Willingness to keep follow up by participants/ relatives.

\section{Exclusion criteria:}

1. Road traffic accident

2. Recurrent stroke

3. Subjects with any recent (6 months) cardiac, orthopaedic, neurological surgeries

4. Subjects with any recent (6 months) cardiac, orthopaedic disorder.

5. Any other progressive/ relapsing neurological disorder like multiple sclerosis, myasthenia gravis, neuropathy, myopathy etc.

\section{Materials}

1. Outcome measures: NIH (National institute of health sciences); FIM (Functional independence measure); SSQOL (Stroke specific quality of life).

2. Patient record sheet for outcome measures.

Study settings: ICU, Physiotherapy OPD, Patient's Home.

Independent variables: Age, Gender

Dependent variable: NIH (National institute of health sciences); FIM (Functional independence measure); SSQOL (Stroke specific quality of life).

Sampling technique: Purposive sampling

Sample size: Statistical considerations: sample size: 234

$\mathrm{N}=(1.96) 2 *(\mathrm{~S} . \mathrm{D}) 2 /$ Permissible error

Permissible error $=15 \%$ of mean $($ mean $=6.4)$

Statistical test: spearman's correlation test

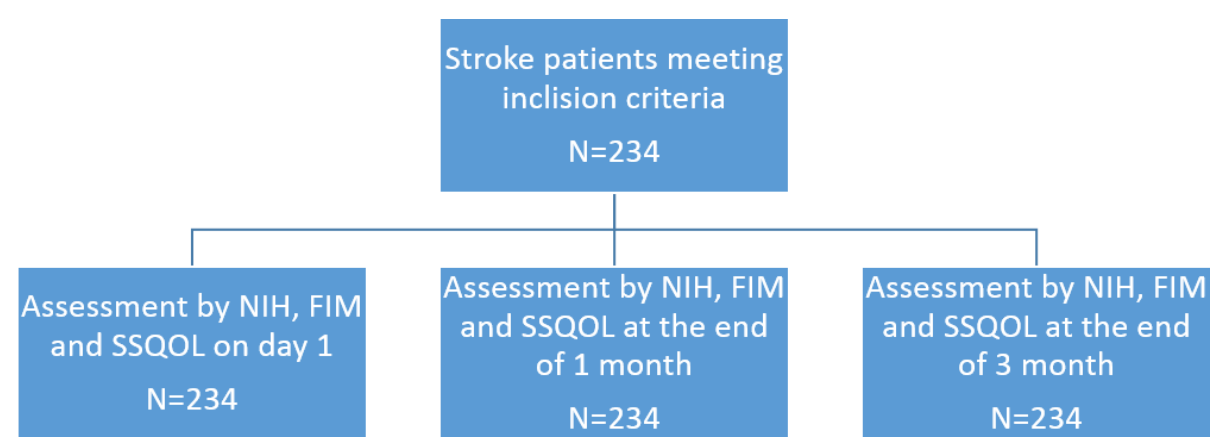

Approval from institutional ethics committee was obtained. Selection of subjects according to inclusion criteria and the procedure was explained. Verbal and written Consent from subject / relatives was taken and the format and details of research work was explained to the patients and the relatives. NIH, FIM and SSQOL scales were evaluated on day 1, at the end of 1 month and at the end of 3 months. Scores were recorded and correlated with each other. Analysis of collected data was done using spearman's correlation. 
Results

Table 1: Shows age wise distribution of the subjects

\begin{tabular}{|l|c|c|}
\hline Age in yrs & No. of cases & \% \\
\hline$<30$ & 7 & 3.0 \\
\hline $30-$ & 11 & 4.7 \\
\hline $40-$ & 37 & 15.9 \\
\hline $50-$ & 58 & 24.9 \\
\hline $60-$ & 65 & 27.9 \\
\hline $70-$ & 41 & 17.6 \\
\hline $80+$ & 14 & 6.0 \\
\hline Total & 233 & 100 \\
\hline
\end{tabular}

Mean Age $=59.4$ years, $\mathrm{SD}=13.8$ years

Table 2

\begin{tabular}{|l|c|c|}
\hline \multicolumn{1}{|c|}{ Gender } & No. of cases & \% \\
\hline Females & 66 & 28.3 \\
\hline Males & 167 & 71.7 \\
\hline Total & 233 & 100 \\
\hline
\end{tabular}

Graph 1: Correlation between NIH and FIM on Day 1

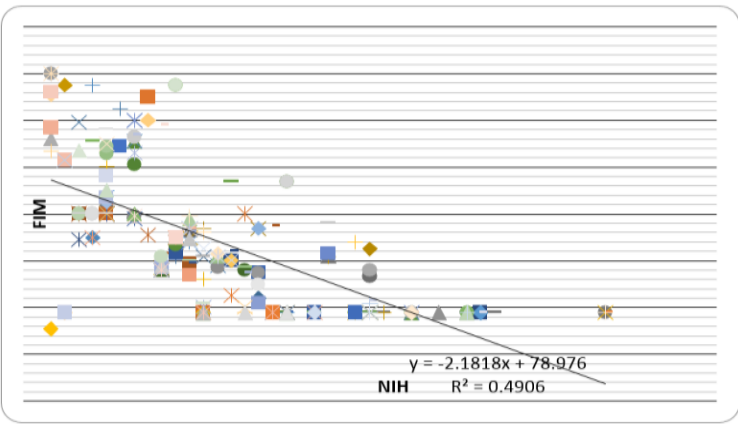

In graph 1 , NIH shows highly significant negative correlation between NIH and FIM on day

Graph 2: Correlation between NIH and SSQOL on Day 1

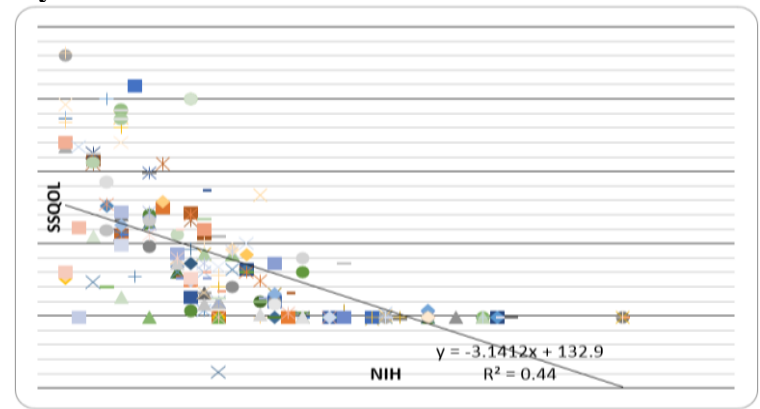

In graph 2, NIH shows highly significant negative correlation with SSQOL on day 1
Graph 3: Correlation between FIM and SSQOL on Day 1

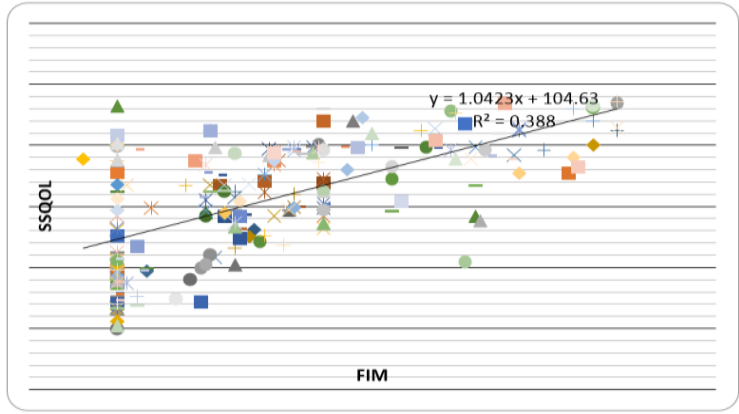

In Graph 3, FIM shows highly significant positive correlation with SSQOL on day 1

Graph 4: Correlation between NIH and FIM at month 1

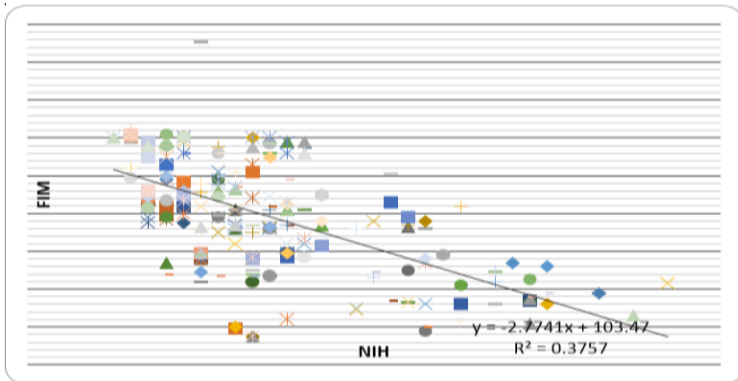

In Graph 4, NIH shows highly significant negative correlation with FIM at the end of 1 month.

Graph 5: Correlation between NIH and SSQOL at month 1

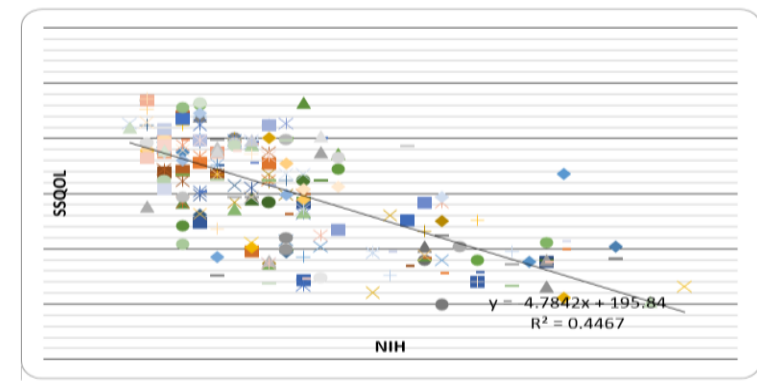

In Graph 5, NIH shows highly significant negative correlation with SSQOL at the end of 1 month. 
Graph 6: Correlation between FIM and SSQOL at month 1

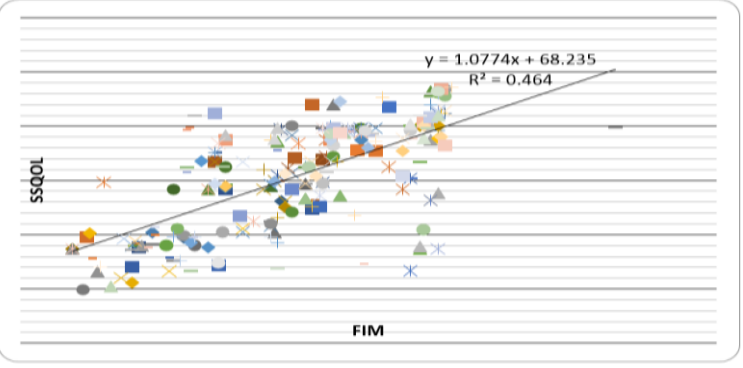

In Graph 6, FIM shows highly significant positive correlation with SSQOL at the end of 1 month.

Graph 7: Correlation between NIH and FIM at month 3

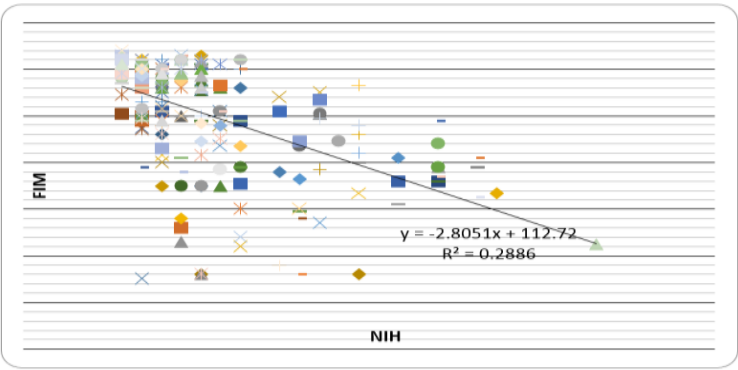

In Graph 7, NIH shows highly significant negative correlation with FIM at the end of 3 months.

Graph 8: Correlation between NIH and SSQOL at month 3

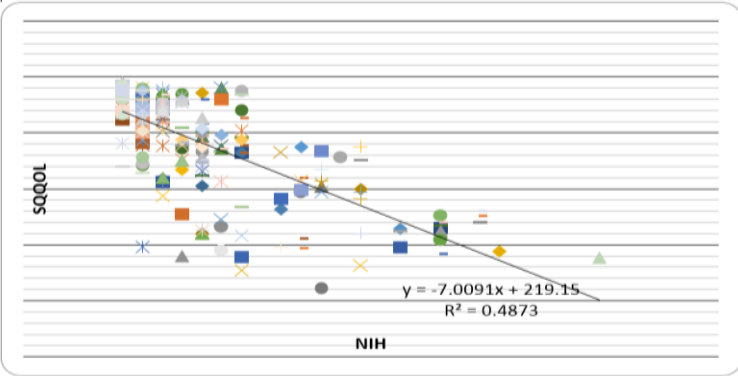

In Graph 8, NIH shows highly significant negative correlation with SSQOL at the end of 3 months.

Graph 9: Correlation between FIM and SSQOL at month 3

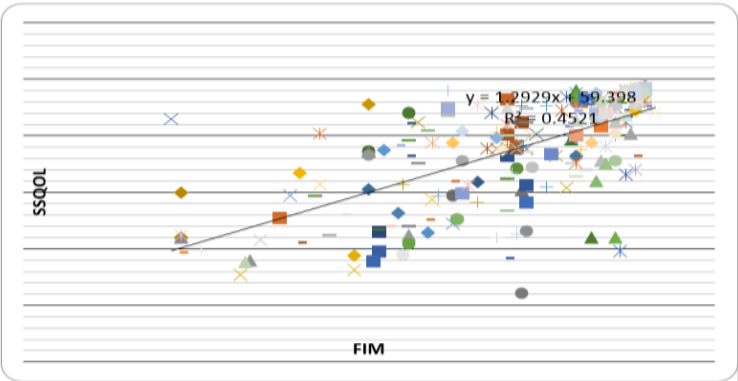

In graph 9, FIM shows highly significant positive correlation with SSQOL at the end of 3 months.

\section{Graph 10: Correlation between NIH-day1 and FIM - month 1}

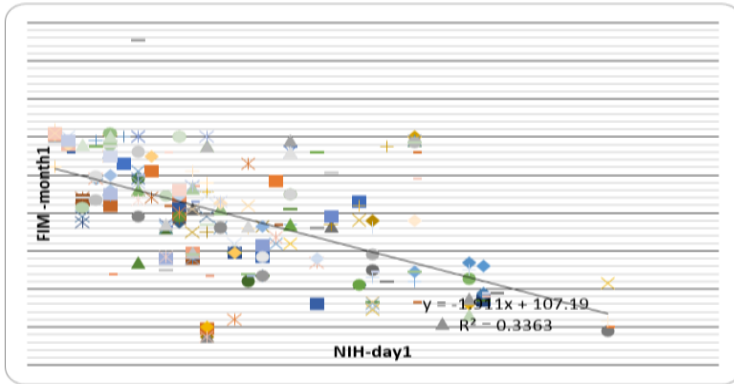

In graph $10, \mathrm{NIH}$ on day 1 shows highly significant negative correlation with FIM at the end of 1 month.

Graph 11: Correlation between NIH-day1 and SSQOL - month1

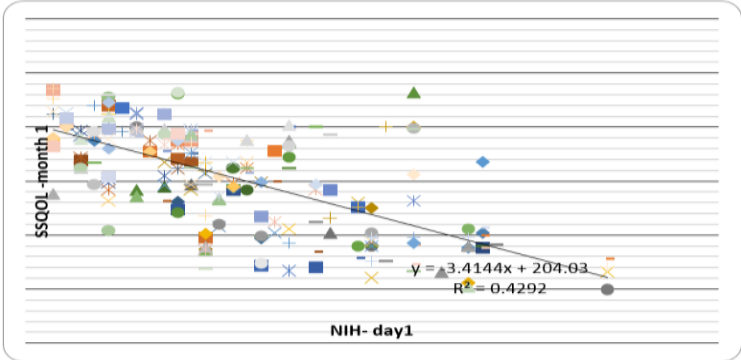

In graph $11, \mathrm{NIH}$ on day 1 shows highly significant negative correlation with SSQOL at the end of 1 month.

\section{Graph 12: Correlation between NIH-day1 and FIM -} month 3

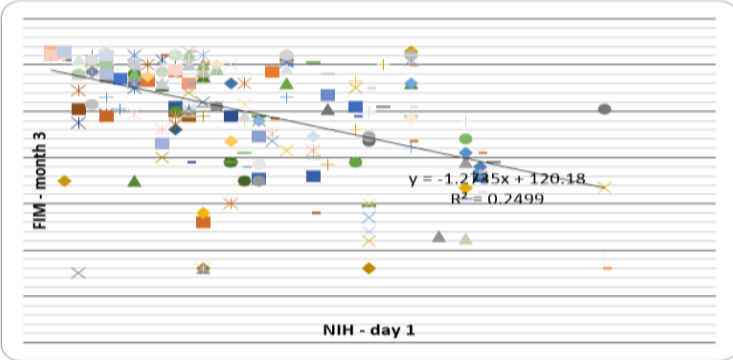

In graph 12 , NIH on day 1 shows highly significant negative correlation with FIM at the end of 3 month 


\section{Graph 13: Correlation between NIH-day1 and} SSQOL - month3

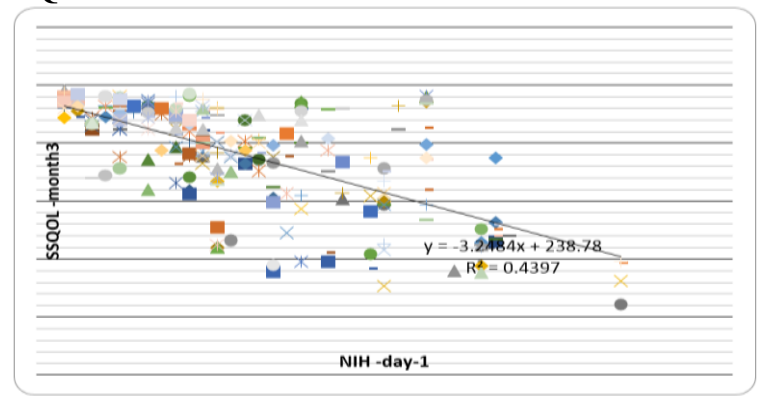

In graph $13, \mathrm{NIH}$ on day 1 shows highly significant negative correlation with SSQOL at the end of 3 months.

\section{Discussion}

An attempt was made to propose a new prognostic study wherein 234 stroke patients were included and were assessed by three outcome measures (NIH which measures severity of stroke, FIM which measures functional limitation and SSQOL which measures quality of life) at three different intervals i.e. on day 1 , at end of 1 month and at end 3. Herein score of NIH taken on day 1, 1 month and 3 months was correlated with FIM and SSQOL score of day 1, FIM score of 1 month, and FIM score of 3 months. Even FIM and SSQOL scores on day 1 , at the end of 1 month and 3 months were correlated with each other.

The result obtained on day 1 of stroke shows that as the NIH score increases, FIM and SSQOL score decreases. That means more the stroke severity, more is the dependency and poor quality of life. On day 1 patient is hospitalized and under supervision so FIM and SSQOL score are always low even though stroke severity of patient is minor, moderate, severe or major. But as the period after stroke increases functional recovery is seen. As per the result obtained at the end of 1 month, it is seen that as the NIH score decreases, FIM and SSQOL score increases to a great extent in case of patient having minor or moderate severity of stroke taken on day 1. Similarly, for end of 3 months, many patients achieve independency and good quality of life if severity of stroke is minor, moderate.

Many patients with stroke experience difficulties due to impairment of the central nervous system. The main purpose of the treatment of stroke is to improve a patient's level of disability to facilitate functional independence, thereby helping to integrate the patient into the local community and improve their QOL. But the improvement also depends upon the severity of stroke. The main criterion for assessing the QOL of patients with stroke is their ability to perform daily living activities independently. FIM has been utilized in a variety of ways to assess the status of the patient and to determine the patient's prognosis and to establish treatment objectives related to the QOL of stroke patients. In this study, the main determinant of QOL and
FIM was severity of stroke. Moderate stroke (low NIH score) would have more affection of functional activities (motor, sensory, speech, transfers) which will further hamper quality of life due to affected socialization, depression, difficulty to speak, transfers, mobility and social cognition which were the most influential of the FIM items affecting QOL. Low score of sensation item on NIH indirectly affects FIM score as perception would be less; patient may have balance issues thereby more risk of fall and might have to use assistive device and patient might refrain themselves from going in outdoors due to fear of fall and thus socialization will be restricted and depression might increase and thus SSQOL score will be low. Individuals with facial impairment (NIH component) can need assistance in eating (FIM component) and can have the communication problems due to reduced facial movements and thus leading to reduced socialization and increase in depression, irritation and change in personality, (SSQOL component). Gaze abnormality (hemianopia) impairment on NIH affects field of vision and require increase in assistance and reduce outdoor mobility which will have a strong impact on socialization, mood swing, depression hampering quality of life (SSQOL). Moderate (Lower NIH score) regarding speech affects the communication and language and has social stigma for individual in society again having a great impact on quality of life. Moderate (Lower score of NIH) motor impairment affects the mobility item of the FIM referring to transfers to bed, chairs, wheelchair, toilet, and bathtub or shower. If patients lack this ability, they are more dependent on caretakers, and thus patient will be homebound and thereby decreasing socialization, increasing depression and mood swings, loneliness hence lowering SSQOL score. Impaired mobility is associated with a loss of independence, reduced QOL, institutionalization, and a higher risk of mortality. Severe and moderate stroke (Lower NIH score) affects the social cognition item on the FIM which refers to social interaction, problem solving, and memory. These items are essential for forming interpersonal relationships, and patients with higher social cognition abilities have better interpersonal relationships. Impairment of these abilities can affect the QOL of patients with stroke. Some items of SSQOL mood, thinking, social roles, personality showed a varied influence on the overall score independent of severity of stroke. Though the severity of stroke is moderate (low NIH score) but if they have problems in the thinking, mood and altered original personality of individual, it might affect the quality of life differently in individuals having same severity of stroke who don't have such problems. It also indirectly affects the motor activities and hampers the FIM score. Accordingly, rehabilitation programs should concentrate on increasing the attention, concentration, information processing capabilities, memory, and judgment ability of patients to improve social cognition. The ability to perform independent activities of daily living is closely 
related to a quick return to social life. Thus, Quality of life, functional activities, and severity of stroke are interrelated and interdependent.

It is now scientifically accepted that the brain does in fact have means of rewiring itself after it has endured a traumatic event; this is where the field of Neuroplasticity gains merit.

Examining the severity and the location of the brain lesion will likely help determine the degree of the deficit that will be procured. Any kind of recovery affects patient quality of life be it neurological or functional. Neurological recovery is defined as recovery of neurological impairments and is often the result of brain recovery/reorganization; it has been increasingly recognized as being influenced by rehabilitation. The majority of neurological recovery occurs within the first 1-3 months. Afterwards recovery may occur much more slowly for up to one year. ${ }^{12}$

Neurological deficits resulting from a stroke are often referred to as impairments. These are determined primarily by the site and extent of the stroke. Most of the spontaneous recovery occurs during the first 3-6 months after the stroke. The course of recovery is a predictable phenomenon; it is initially very rapid and then negatively accelerates as a function of time. The majority of recovery was reported within the first 6 months, recovery continues even after 6 months but it is statistically nonsignificant. ${ }^{12}$

Functional deficits are often referred to as disabilities and are measured in terms of functions such as activities of daily living. Functional recovery is defined as improvement in mobility and activities of daily living; it has long been known that it is influenced by rehabilitation. This recovery depends on the patient's motivation, ability to learn and family supports as well as the quality and intensity of therapy. Functional recovery is influenced by neurological recovery but is not dependent on it. ${ }^{12}$

Reorganization of the brain after a stroke is dependent not only on the lesion site, but also on the surrounding brain tissue and on remote locations that have structural connections with the injured area.

Following a stroke, brain reorganization in response to relearning motor activities, involves primarily the contralateral (affected) hemisphere. Reorganization in response to training occurs along the cortical area of the infarction with increased recruitment of secondary cortical areas such as supplementary motor area and premotor cortex in the contralateral (affected) hemisphere. ${ }^{12}$ Recovery is more rapid and occurs to a greater extent in younger individuals with a stroke and correlates with decline in ability to form neurological connections with aging. There is also a small but significant effect of age on functional recovery. The effect that age has on functional outcomes has been focused on in other rehabilitation research. However, this was not a main component of this research study.
The apparent poorer outcome among patients with hemorrhagic stroke was attributed to greater severity of lesion compared to patients with ischemic stroke. Patients with hemorrhagic strokes have lower functional score upon admission to rehabilitation but tend to be better in terms of functional impairments and achieve higher outcome efficiency scores when compared to those with ischemic strokes. Haemorrhagic stroke patients with the most severely disabling strokes had significantly greater recovery than ischemic strokes of similar severity. However, this was not a main component of this research study.

Actual recovery for each patient could be compared with that predicted by the study, and this information could be used to plan rehabilitation therapy. The comparison of actual and predicted scores could also be used to identify patients who have slow recovering. For these patients, additional intervention may be added.

Although many studies have evaluated outcome after stroke, to the best of knowledge, none has quantified patterns of recovery over time in this way. A trial of physiotherapy after stroke also demonstrated a long-term decline in functional outcome after stroke, which could be reversed by physiotherapy intervention. This study allows the average functional recovery over the entire period up to 3 months after stroke to be quantified. Beyond this point, the FIM and SSQOL should be supplemented with more appropriate measures of long-term recovery, which may be more sensitive to small improvements and to adaptations made by the patient to overcome residual disabilities or impairments if any.

\section{Conclusion}

The results of the study show that there is a highly significant correlation of NIH with FIM and SSQOL. Thus, stroke severity is highly associated with functional outcome and quality of life.

Patient with the stroke severity measurements using NIH score (taken at time of maximum clinical impairment i.e. day 1), can be used to predict the functional limitation of patient and can be useful for the therapist to plan the rehabilitation protocol after stroke. The predicted NIH score of day 1 could be used to plan initial rehabilitation targets, provide patients and their caregivers with some information on the likely pattern of recovery.

Limitations of this study were subjects were not differentiated on basis of type of stroke for statistical analysis. Also the environmental facilitators and barriers were not considered. A similar comparative study can be conducted in patients with and without physiotherapy treatment. A longitudinal study of 6 months to 1 year can also be carried out. 


\section{References}

1. Susan B O'sullivan, Thomas J Schmtiz. Physical rehabilitation $5^{\text {th }}$ edition, New Delhi Jaypee;2007

2. Thom T. Heart Disease and Stroke Statistics--2006

Update: A report from the American Heart Association

Statistics Committee and Stroke Statistics Subcommittee.

Circulation 2006;113(6):e85-e151.

3. Wade (1992) Journal of rehabilitation

Medicine ;Measurement in neurological rehabilitation

4. Sturm JW, Dewey HM, Donnan GA, et al.: Handicap after stroke: how does it relate to disability, perception of recovery, and stroke subtype?: the north North East Melbourne Stroke Incidence Study (NEMESIS). Stroke 2002;33:762-68.

5. Cote R, Hachinski VC. The Canadian Neurological Scale. In: Candelise L, ed. Stroke Scores and Scales. $J$ Cerebrovascular Dis 1992;2:239-47.

6. De Haan R, Limburg M. The Relationship between impairment and functional health scales in the outcome of stroke. Cerebrovasc Dis 1994;4(suppl 2):19-23.

7. Cote R, Hachinski VC, Shurvell BL, Norris JW, Wolfson C. The Canadian Neurological Scale: a preliminary study in acute stroke. Stroke 1986;17:731-37.

8. De Haan R, Horn J, Limburg M, Van Der Meulen J, Bossuyt P. A comparison of five stroke scales with measures of disability, handicap, and quality of life. Stroke 1993;24:1178-11.

9. Keith RA, Granger CV, Hamilton BB, et al.: The functional independence measure: a new tool for rehabilitation. Adv Clin Rehabil 1987,1:6-18.

10. Dickson HG, Kohler F.: Interrater reliability of the 7-level functional independence measure (FIM). Scand J Rehabil Med 1995;27:253-56.

11. Williams LS, Weinberger M, Harris LE, et al. : Development of a stroke-specific quality of life scale. Stroke 1999;30:1362-69.

12. Stroke Rehabilitation Clinician Handbook 2014;3-41. 